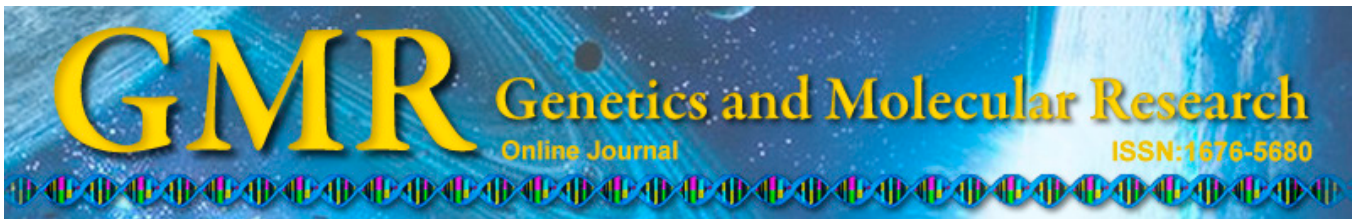

\title{
Genome-wide polymorphisms between the parents of an elite hybrid rice and the development of a novel set of PCR-based InDel markers
}

\author{
K. Wang, J.Y. Zhuang, D.R. Huang, J.Z. Ying and Y.Y. Fan \\ Chinese National Center for Rice Improvement/State Key Laboratory of Rice \\ Biology, China National Rice Research Institute, Hangzhou, China \\ Corresponding author: Y.Y. Fan \\ E-mail: fanyeyangcnrri@163.com
}

Genet. Mol. Res. 14 (2): 3209-3222 (2015)

Received April 8, 2014

Accepted November 24, 2014

Published April 10, 2015

DOI http://dx.doi.org/10.4238/2015.April.10.33

\begin{abstract}
Genome-wide re-sequencing of the Zhenshan 97 (ZS97) and Milyang 46 (MY46) parents of an elite three-line hybrid rice developed in China resulted in the generation of $9.91 \mathrm{G}$ bases of data with an effective sequencing depth of $11.66 \mathrm{x}$ and $11.51 \mathrm{x}$, respectively. Detection of genome-wide DNA polymorphisms, single nucleotide polymorphisms (SNPs), short insertions/deletions (InDels; 1-5 bp), and structural variations (SVs), which is an invaluable variation resource for genetic research and molecular markerassisted breeding, was conducted by comparing whole-genome resequencing data. A total of 364,488 SNPs, 61,181 InDels and 6298 SVs were detected in ZS97 and 364,179 SNPs, 61,984 InDels and 6408 SVs were detected in MY46 compared to the 9311 reference sequence. Synteny analysis of the variation revealed a total of 77,013 identical and 181,737 different SNPs and 15,021 identical and 1205 different InDels between ZS97 and MY46, respectively. A total of
\end{abstract}


180 InDels 3-8 bp in length between ZS97 and MY46 were selected for experimental validation; 160 polymerase chain reaction products were efficiently separated on $6 \%$ non-denaturing polyacrylamide gels. Identification of genome-wide variation among the parents of the elite hybrid as well as the set of 160 polymerase chain reaction-based InDel markers will facilitate future genetic studies and the molecular breeding of hybrid rice.

Key words: Breeding; Hybrid rice; InDels; Polymorphisms; Single nucleotide polymorphisms

\section{INTRODUCTION}

A wide range of naturally occurring variation exists in rice cultivars. These variations of polymorphisms in DNA sequence are the basis of genetic diversity and can be exploited as genetic molecular markers for genetic research and molecular breeding. Numerous types of molecular markers have been developed, such as random amplified polymorphic DNA, amplified fragment length polymorphism, and simple sequence repeats (Jones et al., 2009). Advances in re-sequencing technology have enabled identification of genome-wide genetic variations through large-scale re-sequencing, including millions of single nucleotide polymorphisms (SNPs), the most frequent polymorphism in the genomes of most organisms, and insertions/deletions (InDels) (Shen et al., 2004). The discovery of SNPs in plant and animal gene pools through genome-scale re-sequencing has enabled the development of high-throughput SNP markers and SNP genotyping platforms. Unlike SNPs, which have been studied extensively, other forms of nature genetic variation, such as short InDels (1-5 $\mathrm{bp})$, remain largely undetermined.

China's rice breeders began hybrid development in 1964 using a three-line hybridbreeding system. By 1976, China started large-scale commercial production of the threeline hybrid rice. This technology has contributed to improving food security in China; the increased yield of hybrid rice has helped China feed an extra 60 million people every year ( $\mathrm{Li}$ et al., 2009a). In the three-line hybrid rice-breeding system, the cytoplasmic male sterility line was crossed with the restorer line ( $R$ line) to produce $F_{1}$ hybrid rice, and with the maintainer line (B line) for self-reproduction. Shanyou 10, an elite hybrid that dominated hybrid rice production in South and Central China with 2.35 million hectares of coverage in 1990-2005, was bred from the Zhenshan 97A (ZS97A) and Milyang 46 (MY46) parents (Wan, 2010). These are the most frequently used parents in China, either directly as parents for other hybrids or as crossing donors for parental breeding. ZS97A, an elite cytoplasmic male sterility line, is the most frequently used female parent of widely planted hybrids in China. Zhenshan 97 (ZS97), the B line of ZS97A, is a typical early season indica variety from south China. MY46 is an elite R line derived from a cross involving three International Rice Research Institute varieties (IR8, IR24 and IR262) (Xu et al., 1989). These 2 varieties are representative parents from 2 heterotic pools (early season indica varieties in South China as female parents and low latitude indica varieties from International Rice Research Institute or from other Southeast Asian countries as 
male parent) identified for the three-line hybrid rice in China. Next-generation sequencing technology allowed the discovery of a large number of SNPs and InDels by comparing high-quality re-sequenced whole genome of individuals. Some studies have investigated the genome-wide DNA polymorphisms between indica and japonica cultivars (Shen et al., 2004), among japonica (Yamamoto et al., 2010; Arai-Kichise et al., 2011) or among indica $\mathrm{R}$ lines ( $\mathrm{Li}$ et al., 2012). Until recently, however, little information was available regarding genome-wide polymorphisms between elite hybrid parents, particularly the parents of large-scale planted elite hybrids in China. Further studies examining the genetic diversity of elite hybrid parents can improve our understanding of the 2 heterotic pools of hybrid rice breeding and promote the improvement of $\mathrm{R}$ lines and $\mathrm{B}$ lines for super-hybrid rice breeding.

Many genes/quantitative trait loci (QTL) have been cloned by QTL mapping using indica/indica dual-parent segregation population (Fan et al., 2006; Xue et al., 2008). Indical indica crossing is a common pattern observed in molecular marker-assisted selection (MAS) breeding in indica variety breeding. Limited marker information among indica/indica, however, is a restricting factor in genes/QTL mapping and MAS breeding. To further investigate the utility of short InDels (3-5 bp) among elite three-line hybrid parents in QTL mapping or MAS breeding, we verified the availability of a set of short InDels as a new polymerase chain reaction (PCR)-based molecular marker resource.

The objective of the present study was to identify whole genome-wide DNA polymorphisms between the B line (ZS97) and R line (MY46) of an elite 3-line indica hybrid (Shanyou 10) in China as well as investigating the possible use of short InDels. The wholegenome re-sequencing analysis of ZS97 and MY46 was completed using the Solexa sequencing technology. The sequence-generated reads were mapped to the high-quality 9311 genomic sequence, and genome-wide variations were identified through comprehensive detection of SNPs, InDels, and structural variations (SVs) across the genome. The discovery of these genetic variations provides vital clues for unraveling the genetic basis underlying heterosis in hybrid rice and for improving hybrid rice. A new set of 160 PCR-based short InDel markers developed in this study is a new economically attractive DNA marker resource for genetic research and MAS breeding.

\section{MATERIAL AND METHODS}

\section{Sample preparation and sequencing}

Genomic DNA was extracted from the leaf tissues of an individual plant using a DNeasy Plant Mini Kit (Qiagen, Hilden, Germany). The DNA from each line was then fragmented randomly. After electrophoresis, DNA fragments of the desired length were gel-purified. Adapter ligation and DNA cluster preparation were performed and subjected to Solexa sequencing (Illumina, Inc., San Diego, CA, USA).

\section{Read mapping}

The raw pair-end (PE) sequencing reads were aligned to the 9311 reference genome 
sequence using SOAPaligner ( $\mathrm{Li}$ et al., 2008) using the following criteria: if an original read could not be aligned to the reference sequence, the first base of the 5 '-end and the last 2 bases of the 3'-end were deleted and then realigned to the reference. If alignment could still not be achieved, 2 more bases from the 3 '-end were deleted. This procedure was repeated until the alignment was successful or the modified read was less than $27 \mathrm{bp}$. The average re-sequencing depth and coverage were calculated based on the alignment results.

\section{Assembly of consensus sequences and detection of SNPs}

Based on the alignment results and considering factors such as data characters, sequencing quality, and some other factors from experiments, a Bayesian model was applied to compute the likelihood of genotypes with the actual data. The genotype with the maximum likelihood was selected as the genotype of the re-sequencing individual at a specific base, and a quality value was designated accordingly to reveal the accuracy of the genotype. Polymorphic loci against the reference sequence were selected and filtered if specific criteria were met (e.g., the quality value $>20$ and at least 2 supporting reads) using SOAPsnp (Li et al., 2009b).

\section{Detection of InDels and SVs}

Mapped reads that met the PE requirements and contained gaps at only 1 end were used to detect short InDels $(\leq 5 \mathrm{bp})$. The lengths of the detected InDels were within the range $1-5$ bp. Gaps supported by $\geq 3$ PE reads were retained using SOAPindel (Li et al., 2013). According to the principle of PE sequencing, $1 \mathrm{PE}$ read should be aligned to the forward sequence and another should be aligned to the reverse in normal situations. The distance between the 2 aligned positions on the reference sequence should be in accordance with the size of the insert. If the directions or spans of the alignments of the 2 paired reads were different from what was expected, the region may contain SVs. Abnormal PE alignments observed in our analysis were further analyzed by clustering and compared with previously defined SVs. The SVs were detected using SOAPsv (http://soap.genomics.org.cn) with support from $\geq 3$ abnormal PE reads.

\section{Variations of SNPs and InDels between ZS97 and MY46}

Variations of SNPs and InDels detected for the 2 parental lines were further compared to identify the identical and different SNP/InDel variation. Only variations with $\geq 3$ effective sequence reads were mapped for 2 individuals and selected for further comparison.

\section{Development of short InDel markers}

InDels with insertion/deletion size $\geq 3$ bp between ZS97 and MY46 were selected for further marker design. The Oligo 7.57 software (Molecular Biology Insights, Inc., Cascade, CO, USA) was used to design PCR primers, with a constraint of generating products of 70-180 bp. Genomic DNA was extracted from the leaves of ZS97 and MY46 (Zheng et al., 1995). PCR amplification was conducted as described (Chen et al., 1997). PCR products were separated by electrophoresis ( $6 \%$ non-denaturing polyacrylamide gel) and visualized by silver staining. 


\section{RESULTS}

\section{Genome sequencing}

Whole-genome sequencing was conducted on the genomic DNA of ZS97 and MY46 using the Solexa sequencing technology. Two DNA libraries were constructed and 9.91-G bases were generated. The alignment of reads was used to build consensus genome sequences for each rice accession. Approximately $84.04 \%$ high-quality raw databases were aligned with the reference sequence of the 9311 . The resulting consensus sequence of the 2 rice accessions covered 88.69 and $88.58 \%$ of the reference genome; on average, effective sequencing depths of 11.66x and 11.51x were achieved for ZS97 and MY46, respectively (Table 1 ).

Table 1. Summary of original re-sequencing data.

\begin{tabular}{lcccccc}
\hline Sample & Insert size $(\mathrm{bp})$ & Bases $(\mathrm{G})$ & Mapped bases $(\mathrm{G})$ & Depth $(\%)$ & Coverage $(\%)$ & Mismatch rate $(\%)$ \\
\hline ZS97 & 477 & 4.98 & 4.19 & 11.66 & 88.69 & 0.77 \\
MY46 & 472 & 4.93 & 4.14 & 11.51 & 88.58 & 0.66 \\
\hline
\end{tabular}

\section{Identification and distribution of variation across the rice genome}

A total of 364,488 SNPs, 61,181 InDels, and 6298 SVs were detected between ZS97 and the 9311 reference sequence; 364,179 SNPs, 61,984 InDels, and 6408 SVs were detected between MY46 and the 9311 reference sequence (Table 2). The total polymorphisms detected in the 2 parental lines varied across different chromosomes. The largest number of polymorphisms $(52,639$ and 56,817) was observed on chromosomes 1 and 5 for ZS97 and MY46, respectively, while chromosomes 5 and 11 showed the lowest number of polymorphisms for ZS97 and MY46, respectively.

The genomic distribution of DNA polymorphisms (SNPs, InDels, and SVs) between the 2 accessions and the 9311 reference genome were examined by calculating the frequency of polymorphisms observed for each 500-kb interval along the chromosome. The average density of DNA polymorphisms detected per $500 \mathrm{~kb}$ across the genome in ZS97 and MY46 were similar to the 486.6 and 486.2 SNPs, 81.7 and 82.7 InDels, and 8.4 and 8.6 SVs, respectively (Table 2 and Figure 1). The frequency of SNPs within the genome with chromosomes 11 and 5 showed the highest (721.0) and the lowest densities (368.7) per 500-kb interval in ZS97, while chromosomes 11 and 5 showed the lowest (313.0) and the highest densities (765.0) per 500-kb interval in MY46; similar frequency results with SNPs were observed for InDels. The frequency of SVs within the genome with chromosomes 11 and 7 showed the highest (12.0) and the lowest densities (5.7) in ZS97, and MY46 showed chromosomes 5 and 2 with the highest (11.5) and the lowest densities (6.0) (Table 2).

Of the total short InDels (1-5 bp) detected between samples and the 9311 reference sequence, 30,411 insertions and 30,770 deletions were detected in ZS97 and 30,920 insertions and 31,064 deletions were detected in MY46. A skewed distribution was observed for InDel length, and most (72.5 and $72.1 \%$ ) were mononucleotides, while 11.6 and $12.0 \%$ were $3-5$-bp insertions or deletions in ZS97 and MY46, respectively (Table 3). 


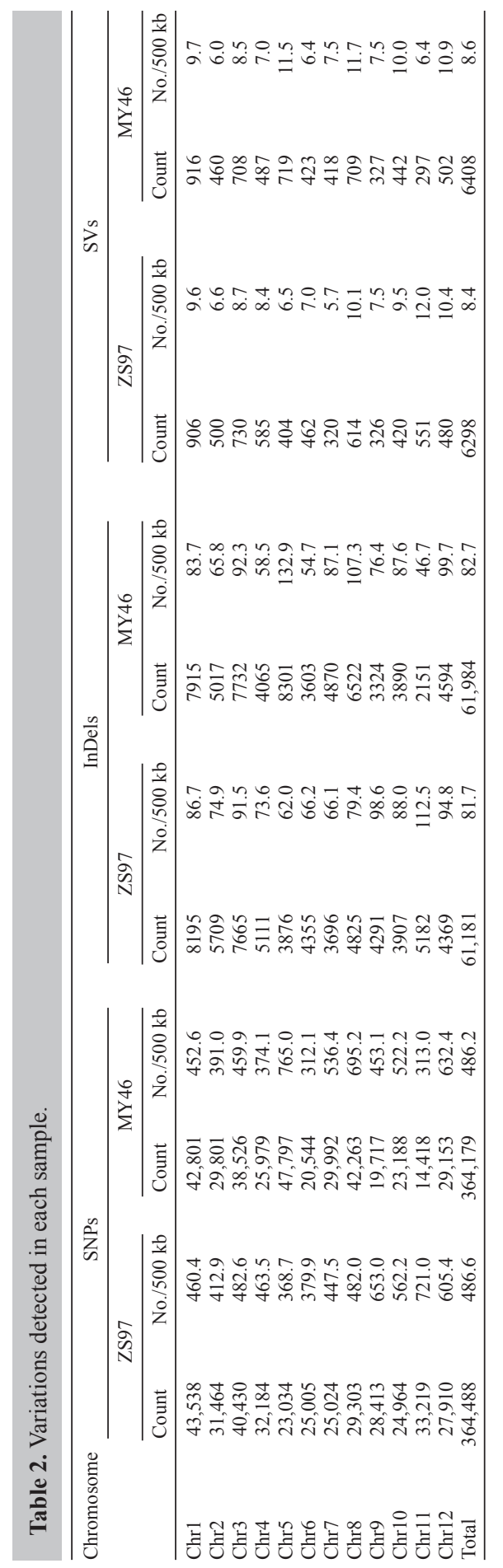




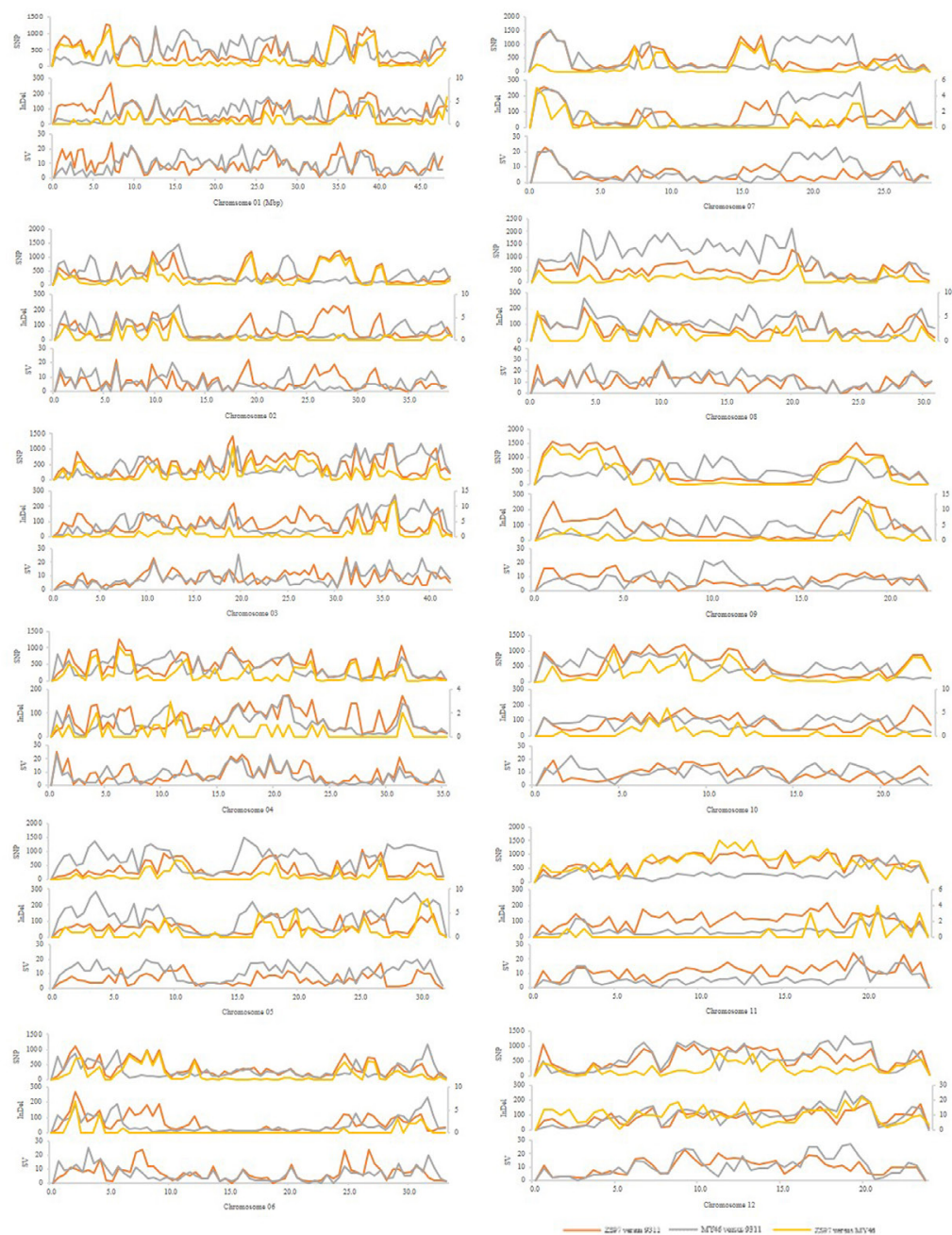

Figure 1. Distribution of DNA polymorphisms identified between 9311, ZS97 and MY46 along each chromosome. The $\mathrm{x}$-axis represents the physical distance along each chromosome, split into 500-kb intervals. The first y-axis indicates the number of three kinds of DNA polymorphisms and the second y-axis indicates the number of InDel polymorphisms between ZS97 and MY46.

Table 3. Number of short InDels identified in the two re-sequencing samples.

\begin{tabular}{lcrr}
\hline InDel size $(\mathrm{bp})$ & ZS97 vs 9311 & MY46 vs 9311 & ZS97 vs MY46 \\
\hline 1 & 44,371 & 44,689 & 612 \\
2 & 9714 & 9867 & 413 \\
3 & 3675 & 3822 & 117 \\
4 & 2388 & 2539 & 39 \\
5 & 1033 & 1067 & 11 \\
6 & 0 & 0 & 12 \\
7 & 0 & 0 & 0 \\
8 & 0 & 0 & 1 \\
Total & 61,181 & 61,984 & 1205 \\
\hline
\end{tabular}




\section{Genetic variation between ZS97 and MY46}

DNA variations between ZS97 and MY46 may reflect the basis of genetic divergence of B lines and R lines of three-line hybrids. Synteny analysis of the variations revealed a total of 77,013 identical SNPs and 181,737 different SNPs as well as 15,021 identical InDels and 1205 different InDels between ZS97 and MY46 (Table 4 and Table S1). The frequency of SNPs between ZS97 and MY46 varied from 283.1 SNPs/Mb on chromosome 8 to 984.7 $\mathrm{SNPs} / \mathrm{Mb}$ on chromosome 11 , with an average of $485.2 \mathrm{SNPs} / \mathrm{Mb}$; the frequency of InDels between ZS97 and MY46 varied from 1.4 InDels/Mb on chromosome 4 to 5.9 InDels/Mb on chromosome 12, with an average of $3.2 \mathrm{InDels} / \mathrm{Mb}$ (Table 4).

Table 4. Variations detected between the two re-sequencing samples.

\begin{tabular}{|c|c|c|c|c|c|c|}
\hline \multirow[t]{2}{*}{ Chromosome } & \multicolumn{3}{|c|}{ SNPs } & \multicolumn{3}{|c|}{ InDels } \\
\hline & No. identical & No. different & No. different/Mb & No. identical & No. different & No. different/Mb \\
\hline Chr1 & 9231 & 22,978 & 486.0 & 1921 & 157 & 3.3 \\
\hline $\mathrm{Chr} 2$ & 4281 & 18,282 & 479.8 & 1110 & 92 & 2.4 \\
\hline $\mathrm{Chr} 3$ & 9464 & 20,638 & 492.7 & 2001 & 180 & 4.3 \\
\hline Chr4 & 8203 & 14,283 & 411.4 & 1609 & 48 & 1.4 \\
\hline Chr5 & 5013 & 8890 & 284.6 & 1046 & 153 & 4.9 \\
\hline Chr6 & 3330 & 13,080 & 397.4 & 926 & 72 & 2.2 \\
\hline $\mathrm{Chr} 7$ & 6091 & 10,261 & 367.0 & 1069 & 56 & 2.0 \\
\hline Chr8 & 9863 & 8605 & 283.1 & 1682 & 120 & 3.9 \\
\hline Chr9 & 3785 & 18,452 & 848.1 & 723 & 89 & 4.1 \\
\hline Chr10 & 6436 & 11,949 & 538.1 & 1107 & 65 & 2.9 \\
\hline Chr11 & 3342 & 22,682 & 984.7 & 690 & 38 & 1.6 \\
\hline Chr12 & 7974 & 11,637 & 504.9 & 1137 & 135 & 5.9 \\
\hline Total & 77,013 & 181,737 & 485.2 & 15,021 & 1205 & 3.2 \\
\hline
\end{tabular}

Of the total 1205 different InDels detected between ZS97 and MY46, length varied in the range of 1-8 bp, indicating bias towards mononucleotide InDels, most (50.8\%) of which were mononucleotides, $34.3 \%$ were of 2 bp variation, and $14.9 \%$ were of $3-8$ bp variation (Table 3 ). InDels $\geq 3 \mathrm{bp}$, which can be observed easily on polyacrylamide gel electrophoresis, are potential variations that can be used for PCR-based marker design.

\section{Potential of short InDels as PCR-based markers}

Whole-genome sequencing technology allowed the detection of InDels as well as of SNPs. To validate the availability of InDels identified between the two 3-line restorer and maintainer accessions as novel PCR-based DNA markers, we selected short InDels $\geq 3$ bp and converted them to PCR-based markers. A total of $180 \mathrm{InDels}$ (3-8 bp), which were distributed across the 12 chromosomes varying from 30 InDels on chromosome 3 to 6 InDels on chromosomes 7 and 11, were selected between the ZS97 and MY46 genomes. Based on this selection, we designed primer pairs to amplify $70-176$ bp surrounding the InDels. Following PCR analysis, all 180 primer pairs showed reliable amplification using genomic DNA of ZS97 and MY46 as the DNA template; 160 of the InDels (88.9\%) identified were polymorphic between ZS97 and MY46 as determined using electrophoresis (6\% non-denaturing polyacrylamide gel) and 20 were non-significant polymorphisms [Table 5, Table S2 and Figure S1 (A-L)]. 


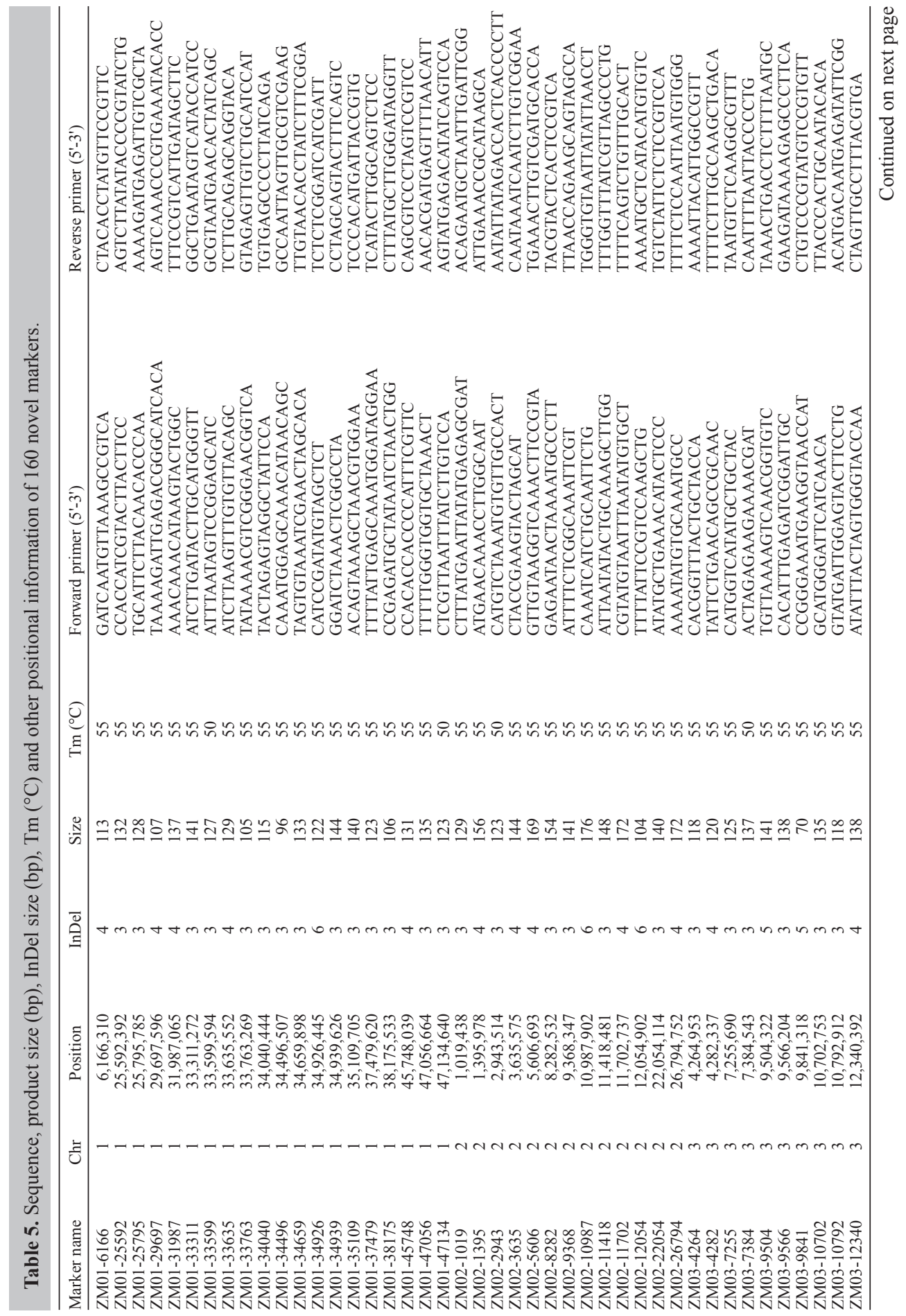




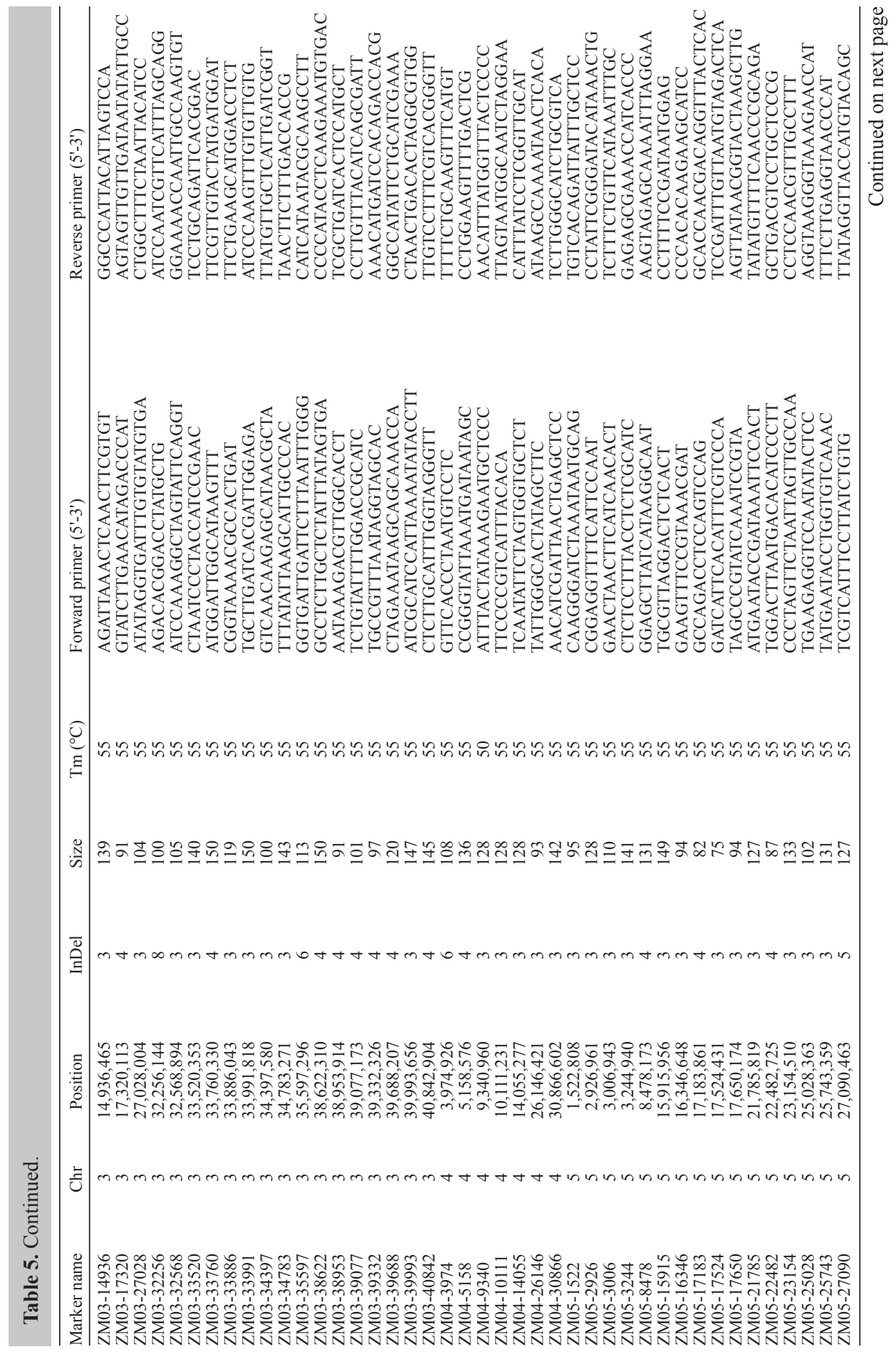




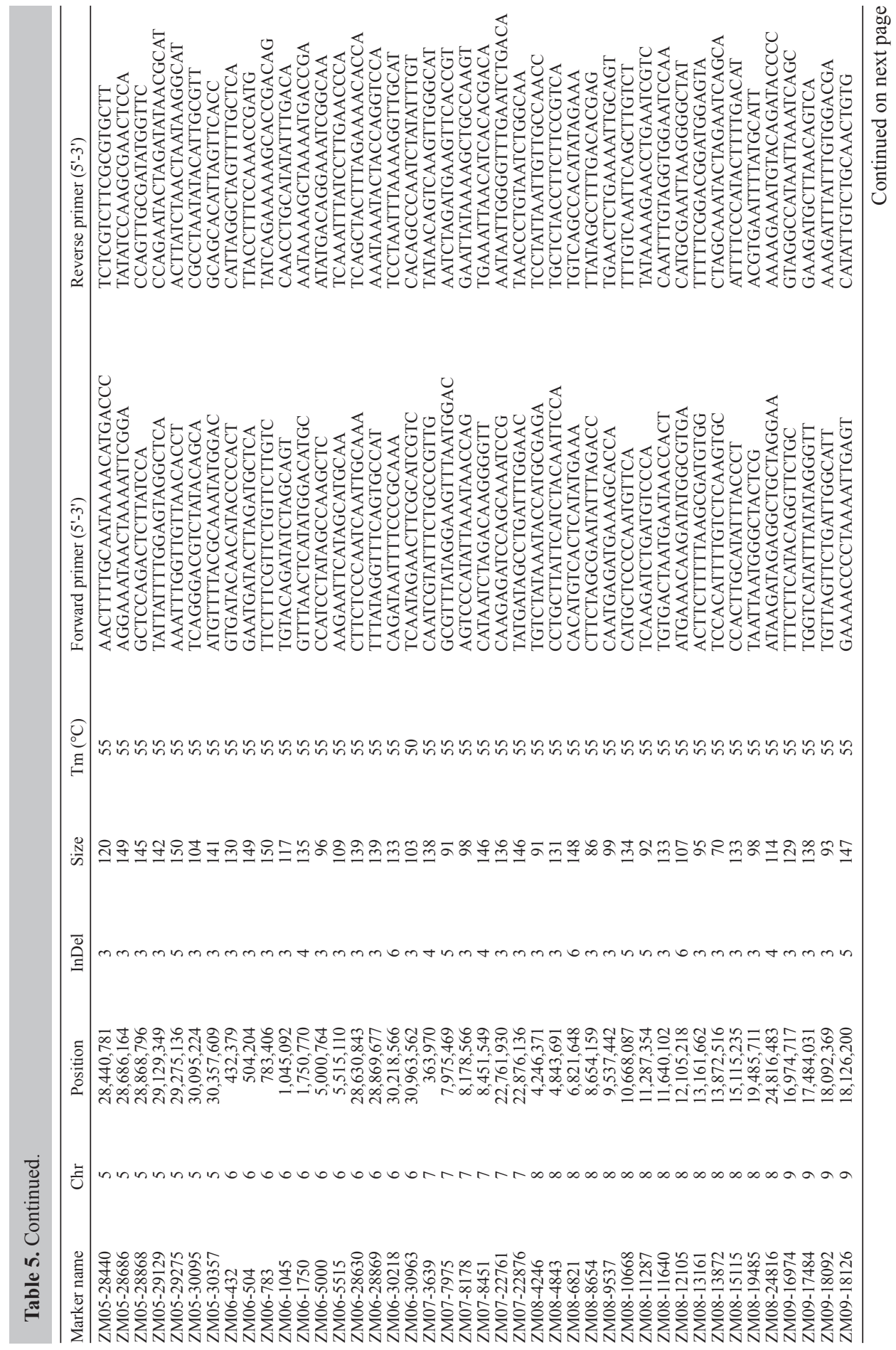




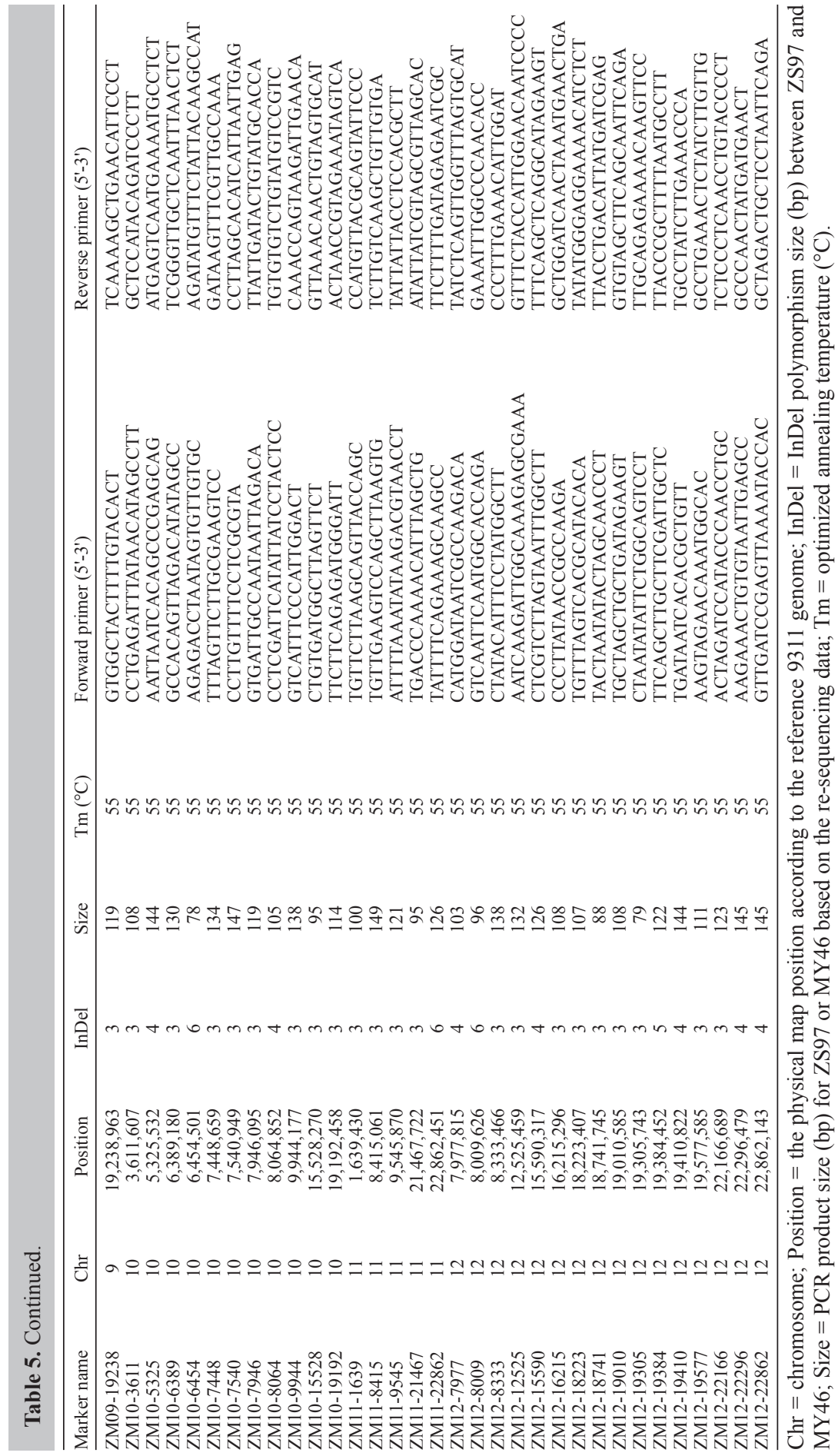




\section{DISCUSSION}

Genetic diversity within parental lines of hybrid rice is the foundation of heterosis utilization in hybrid rice breeding. In the present study, genome analysis based on re-sequencing of 2 parental lines, ZS97 and MY46, of an elite 3-line indica hybrid was used for comprehensive identification of SNP, InDel, and SV variations. This information provides valuable clues for understanding heterosis and improving hybrid rice breeding.

Compared to the 9311 reference sequence, a total of 364,488 SNPs, 61,181 InDels, and 6298 SVs were detected in ZS97; 364,179 SNPs, 61,984 InDels, and 6408 SVs were detected in MY46. Additionally, 77,013 identical SNPs and 181,737 different SNPs as well as 15,021 identical InDels and 1205 different InDels were observed between ZS97 and MY46. The total number of different SNPs and InDels estimated between ZS97 and MY46 was much lower compared to that reported for more diverse landrace populations, as well as between indica and japonica cultivars (Shen et al., 2004; Huang et al., 2010; Subbaiyan et al., 2012). This may be because of the absence of SNPs/InDels from 1 sample, resulting in lower re-sequencing depth and coverage, as well as the strict filter requirement (at least 3 supporting reads for every variation in both 2 samples) in comparative analysis and the closed relationship of the 2 samples. However, the total genetic variation detected between ZS97 (B line) and MY46 (R line) was higher compared to the $\mathrm{R}$ lines of cytoplasmic male sterility and among japonica (Yamamoto et al., 2010; Arai-Kichise et al., 2011; Li et al., 2012), which is consistent with the expected genetic divergence between the $\mathrm{B}$ and $\mathrm{R}$ lines of three-line hybrid and was higher compared to $\mathrm{R}$ lines. These variations improve the understanding of the complicated genetic basis of the 2 heterotic pools of the three-line hybrid rice in China.

Simple sequence repeat and SNP systems are the 2 most widely used markers in genetic research and MAS breeding of rice. Because of the limited number and nonfunctional variation of simple sequence repeat and strict platform requirement for SNP detection, InDels have become a valuable resource for genetic research (Shen et al., 2004; Subbaiyan et al., 2012). InDels with moderate size differences are preferable for their ease of genotyping and have been used widely in genes/QTL mapping (Zhang et al., 2012) and rice MAS breeding (Hayashi et al., 2006). There are 2 subspecies of cultivated rice, indica and japonica, and most early development of InDel markers was based on sequence comparison between the 2 subspecies with distant genetic divergence (Shen et al., 2004; Wang et al., 2005); most of these InDel markers cannot reflect the genetic divergence within subspecies. Recent advance in next-generation of re-sequencing technology have made it possible to identify InDel markers within the same subspecies and even accessions with close genetic relationships. Both increased and saturated availability of DNA markers between indica cultivars are vitally important for efficient 3-line indica hybrid breeding and genetic research based on genetic populations derived from indicalindica crosses. In our study, of 1205 different short InDels between ZS97 and MY46, 180 were 3-8 bp in length, which was easily detected by polyacrylamide gel electrophoresis, and may be the preferred method for developing PCR-based markers. We converted these InDels into PCR-based markers; 160 of the tested InDels produced amplified fragments and showed polymorphisms between ZS97 and MY46, which can be used as a new source of markers for genetic studies based on indicalindica-derived populations and molecular improvement of three-line hybrid rice breeding. 


\section{ACKNOWLEDGMENTS}

Research supported by the China National High-Tech Research and Development Program (\#2012AA101102), the Chinese High-Yielding Transgenic Program (\#2013ZX08001004), and the Research on Key Teamwork Construction in Technology Innovations of Zhejiang Province, China (\#2010R50024).

\section{Supplementary material}

\section{REFERENCES}

Arai-Kichise Y, Shiwa Y, Nagasaki H, Ebana K, et al. (2011). Discovery of genome-wide DNA polymorphisms in a landrace cultivar of japonica rice by whole-genome sequencing. Plant Cell Physiol. 52: 274-282.

Chen X, Temnykh S, Xu Y, Cho YG, et al. (1997). Development of a microsatellite framework map providing genomewide coverage in rice (Oryza sativa L.). Theor. Appl. Genet. 95: 553-567.

Fan C, Xing Y, Mao H, Lu T, et al. (2006). GS3, a major QTL for grain length and weight and minor QTL for grain width and thickness in rice, encodes a putative transmembrane protein. Theor. Appl. Genet. 112: 1164-1171.

Hayashi K, Yoshida H and Ashikawa I (2006). Development of PCR-based allele-specific and InDel marker sets for nine rice blast resistance genes. Theor. Appl. Genet. 113: 251-260.

Huang X, Wei X, Sang T, Zhao Q, et al. (2010). Genome-wide association studies of 14 agronomic traits in rice landraces. Nat. Genet. 42: 961-967.

Jones N, Ougham H, Thomas H and Pasakinskiene I (2009). Markers and mapping revisited: finding your gene. New Phytol. 183: 935-966.

Li J, Xin Y and Yuan L (2009a). Hybrid rice technology development: Ensuring China's food security. International Food Policy Research Institute (IFPRI), Washington, DC.

Li R, Li Y, Kristiansen K and Wang J (2008). SOAP: short oligonucleotide alignment program. Bioinformatics 24: 713-714.

Li R, Li Y, Fang X, Yang H, et al. (2009b). SNP detection for massively parallel whole-genome resequencing. Genome Res. 19: 1124-1132.

Li S, Wang S, Deng Q, Zheng A, et al. (2012). Identification of genome-wide variations among three elite restorer lines for hybrid-rice. PloS One 7: e30952.

Li S, Li R, Li H, Lu J, et al. (2013). SOAPindel: Efficient identification of indels from short paired reads. Genome Res. 23: $195-200$

Shen Y, Jiang H, Jin J, Zhang Z, et al. (2004). Development of genome-wide DNA polymorphism database for map-based cloning of rice genes. Plant Physiol. 135: 1198-1205.

Subbaiyan GK, Waters DL, Katiyar SK, Sadananda AR, et al. (2012). Genome-wide DNA polymorphisms in elite indica rice inbreds discovered by whole-genome sequencing. Plant Biotechnol. J. 10: 623-634.

Wan JM (2010). Chinese rice genetics and breeding and breed pedigree: 1986-2005. China Agricultural Press, Beijing (in Chinese)

Wang X, Zhao X, Zhu J and Wu W (2005). Genome-wide investigation of intron length polymorphisms and their potential as molecular markers in rice (Oryza sativa L.). DNA Res. 12: 417-427.

Xu XZ, Chen KR, Tong HJ and Cai QH (1989). The development and utilization of restorer line Milyang 46. Hybrid Rice 4: 22-24 (in Chinese).

Xue W, Xing Y, Weng X, Zhao Y, et al. (2008). Natural variation in $G h d 7$ is an important regulator of heading date and yield potential in rice. Nat. Genet. 40: 761-767.

Yamamoto T, Nagasaki H, Yonemaru J, Ebana K, et al. (2010). Fine definition of the pedigree haplotypes of closely related rice cultivars by means of genome-wide discovery of single-nucleotide polymorphisms. BMC Genomics 11: 267.

Zhang Z, Wang K, Guo L, Zhu YJ, et al. (2012). Pleiotropism of the photoperiod-insensitive allele of Hdl on heading date, plant height and yield traits in rice. PloS One 7: e52538.

Zheng KL, Huang N, Bennett J and Khush GS (1995). PCR-based marker-assisted selection in rice breeding. IRRI Discussion Paper Series No. 12. International Rice Research Institute, Los Baños. 